\title{
Imaginología por resonancia magnética y la determinación de cloruro y sodio en mortero de cemento Pórtland
}

\author{
P.F. de J. Cano-Barrita ${ }^{1}$, B. J. Balcom ${ }^{2}$ y T.W. Bremner ${ }^{3}$ \\ 'CIIDIR-Oaxaca, Instituto Politécnico Nacional de México, Oaxaca. \\ ${ }^{2}$ MRI Centre, Physics Department, University of New Brunswick, Fredericton, NB, Canada \\ ${ }^{3}$ Civil Engineering Department, University of New Brunswick, Fredericton, NB, Canada \\ E-mails: pcano@ipn.mx,bjb@unb.caybremnert@unb.ca
}

(Recibido: marzo de 2006; aceptado: diciembre de 2006)

\section{Resumen}

La corrosión del acero de refuerzo es una de las causas principales del deterioro de estructuras de concreto. La penetración de cloruros a través de difusión o absorción capilar o una combinación de ambos, contribuye de manera importante en la corrosión del acero de refuerzo. En países con clima frío, las sales descongelantes usadas en invierno son un factor importante en el deterioro de estructuras, y en muchos países, los cloruros del agua de mar exacerban el proceso de deterioro. Para evaluar la distribución de cloruros en concreto, normalmente se utilizan métodos destructivos, cuya resolución espacial es baja y consumen una cantidad consid er able de tiempo. Una alternativa no destructiva es el uso de la técnica SPRITE de resonancia magnética, la cual ha demostrado ser conveniente para determinar la distribución de diferentes especies en materiales basados en cemento Pórt land. En este artículo se demostrará la habilidad de la técnica SPRITE para determinar la distribución de cloruros y sodio en mortero, durante absorción capilar y difusión. Además, los experimentos preliminares indican que la técnica puede ser utilizada para determinar la distribución de cloruros en mortero y concreto, conteniendo cemento ordinario. Esto abre la posibilidad de estudiar la distribución de cloruros en corazones de concreto extraídos de estructuras reales.

Descriptores: Resonancia magnética, cloruros, no destructivo, difusión, absorción capilar, mortero.

\footnotetext{
Abstract

Cor ro sion of the re in force ment is one of the main causes of de te rio ra tion of con crete structures. Chloridepenetration intoconcretedue toeitherdiffusionorabsorp tion isan im por tant con trib u tor to the cor ro sion of em bed ded steel in con crete. In northern countries, deicing salts are a ma jor fac tor in the de te ri o ra tion of high ways and in all countries, chloridesfrom seawaterexacer bate the deterioration process. Inorder toassess the chloridepene tration, destructive, lowspatial resolu tionand time consuming methods are rou tinely used. The SPRITE Mag netic Res o nance Im aging is a non-destructive tech nique that has dem on strated to be suit able to image ma te rials pos sess ing short lifetime sig nals in clud ing Pórt land ce ment based ma te rials. This pa per will dem on strate the abil ity of SPRITE MRI to de ter mine the dis tribu tion of chlo rides in mor tar dur ing $\mathrm{NaClsolutioncapillaryabsorptionordiffusion}$. Inaddition, preliminaryexperiments
} 
Imaginología por resonancia magnética y la determinación de cloruro y sodio en mortero de ...

in di cate that the tech nique can be used to im age the dis tri bu tion of chlo rides in concrete/mor tar con tain ing nor mal Port land ce ment, which makes it pos sible to study the pen e tra tion of chlo rides in cores taken from field con crete.

Keywords:Magnetic res onance, chlo rides, non-destructive, diffusion, cap il laryabsorption, mortar.

\section{Introducción}

La determinación de la profundidad de penetración y distribución de cloruros en materiales basados en cemento Pórtland, es importante desde el punto de vista de durabilidad de estructuras de concreto reforzado.

El ingreso de cloruros, a través de procesos de difusión y absorción capilar, puede depasivar la capa protectora sobre el acero de refuerzo y causar corrosión del mismo.

La manera usual de determinar la profundidad de penetración o la distribución de cloruros en concreto, implica destruir el espécimen. Las técnicas comunes requieren obtener muestras de polvo a diferentes profundidades y mediante procedimientos de titulación química se determina la concentración de cloruros. Otra prueba sólo requiere aplicar una solución (por ejemplo, nitrato de plata) sobre un espécimen recién fracturado para determinar la profundidad de penetración de cloruro (Otsuki et al., 1992). El primer procedimiento proporciona un perfil con resolución espacial baja y es inherentemente destructivo. Sin embargo, con dispositivos adecuados es posible aumentar la resolución al obtener muestras de polvo a distancias de unos cuantos milímetros, aunque el problema de destruir el espécimen persiste. El segundo procedimiento sólo proporciona la profundidad de penetración de los cloruros y el perfil de concentración se desconoce.

La resonancia magnética ha sido utilizada con éxito para determinar en forma no destructiva la distribución de agua, cloruros y sodio en materiales de construcción. Pel et al. (2000), realizaron un estudio de la penetración de solución de $\mathrm{NaCl}$ en ladrillo de silicato de calcio; sin embargo, la técnica tradicional de resonancia magnética utilizada tiene limitaciones en cuanto a la vida de la señal, $\mathrm{T}_{2}^{*}$, y sólo pudieron obtenerse perfiles de sodio y agua. Cano et al. (2002) obtuvieron los primeros perfiles de cloruros, sodio y agua en mortero de cemento portland blanco durante absorción capilar de una solución de $\mathrm{NaCl}$. En esta investigación se utilizó cemento Pórtland blanco, debido a su bajo contenido de hierro, lo cual era necesario para determinar la distribución de agua, pero normalmente el utilizado en la práctica contiene cemento Pórtland ordinario y adiciones minerales (ceniza volante, humo de sílice, escoria de alto horno, etc.) que incrementan sustancialmente la cantidad de impurezas paramagnéticas en la muestra.

Este artículo presentará los resultados del uso de la técnica de resonancia magnética SPRITE para determinar la distribución de cloruros y sodio en mortero que contiene cemento Pórtland ordinario durante absorción capilar de una solución de $\mathrm{NaCl}$. También se determinó la distribución de cloruro y sodio durante difusión. Es importante mencionar que el uso de materiales con bajo contenido de hierro no es necesario en el caso de ${ }^{35} \mathrm{Cl}$ y ${ }^{23} \mathrm{Na}$, como en la determinación de la distribución de ${ }^{1} \mathrm{H}$ del agua. Esto significa que en concreto el fabricado con varios tipos de cemento Pórtland, la mayoría de los tipos de agregados pueden ser utilizados. 


\section{Técnica Sprite (Single Point Ramped Imaging With $T_{1}$ Enhancement)}

Detalles de la técnica SPRITE pueden obtenerse en Balcomet al. (1996). La intensidad de la señal, $S$, en cualquier punto de una imagen uni-dimensional (perfil), se relaciona con la densidad de núcleos local, por medio de la ecuación (1).

$$
S=\rho \cdot e^{-\frac{t p}{T_{2} *}} \cdot\left(\frac{1-e^{-\frac{T R}{T_{1}}}}{1-\cos \alpha \cdot e^{-\frac{T R}{T_{1}}}}\right) \cdot \sin \alpha
$$

donde tp es el tiempo de codificación de la fase, $\mathrm{T}_{2}{ }^{*}$ es el tiempo de relajación "spinspin", TR es el tiempo de la repetición de los pulsos de radio frecuencia, $\alpha$ es el ángulo de rotación de la magnetización y T1 es el tiempo de relajación "spin-lattice". En la ecuación (1) el término entre paréntesis representa el estado constante de la magnetización longitudinal establecido después de varios pulsos de radio frecuencia y es válido sólo para el hidrógeno del agua que tiene "spin" I=1/2. Este término tendrá una representación más compleja para el sodio y núcleos del cloro que tienen "spin" I=3/2 (Mitrovic et al., 2001). Esta técnica se representa esquemáticamente en la figura 1.

\section{Procedimiento experimental}

En la preparación de morteros con relación $a / c=0.60$ se utilizó agregado de cuarzo, cemento Pórtland blanco y ordinario Tipo I. En el cuadro 1 se proporcionan las propiedades físicas y químicas de los cementos usados. Se fabricaron cilindros de $30 \mathrm{~mm}$ de diámetro y se curaron en húmedo durante tres días a $38^{\circ} \mathrm{C}$. Después, los especimenes se cortaron a una longitud de $30 \mathrm{~mm}$ y se secaron en horno a $105^{\circ} \mathrm{C}$ hasta masa constante. Finalmente, en la superficie cilíndrica se aplicó resina epóxica para hacer la penetración de la solución de $\mathrm{NaCl}$ uniaxial (Figura 2).

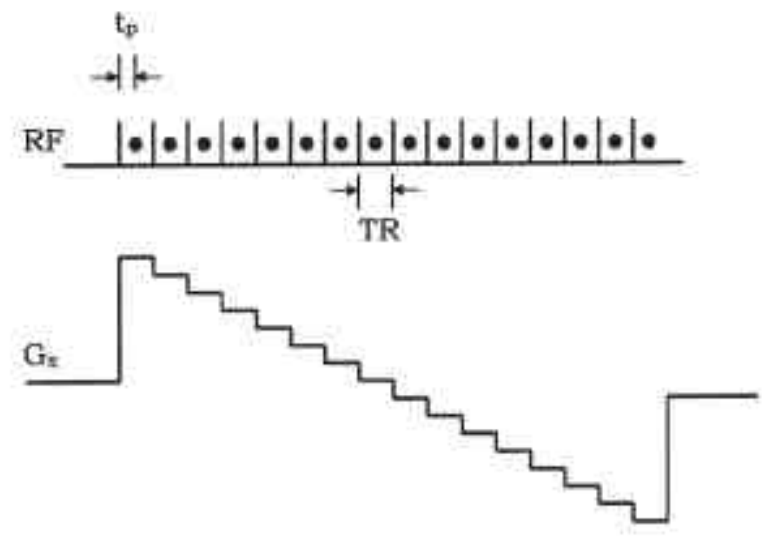

Figura 1. La técnica SPRITE uni-dimensional. El gradiante del campo magnético es cambiado en 64 pasos. Solo un punto es adquirido en cada cambio del gradiante a un tiempo tp después de la aplicación de cada pulso de radio de frecuencia de radio, con ángulo $\alpha$. El tiempo de repetición, TR, es el tiempo entre

la aplicación de pulsos de radio frecuencia en cada cambio de gradiente 
Imaginología por resonancia magnética y la determinación de cloruro y sodio en mortero de ...

Cuadro 1. Propiedades físicas y químicas del cemento ordinario y blanco

\begin{tabular}{|c|c|c|}
\hline & $\begin{array}{l}\text { Cemento } \\
\text { ordinaino Tipo } 1\end{array}$ & $\begin{array}{l}\text { Cemiento } \\
\text { blanco Tipo } 1\end{array}$ \\
\hline \multicolumn{3}{|l|}{ Propiedades fisicas } \\
\hline Gravedad especifica & 315 & 315 \\
\hline Superficie epecifica, Blaine, $\left(\mathrm{m}^{2} / \mathrm{kg}\right)$ & 388 & 404 \\
\hline \multicolumn{3}{|l|}{ Anâlisis quimico (\% en masa) } \\
\hline $\mathrm{SiO}_{2}$ & 20,6 & 22.45 \\
\hline $\mathrm{Al}_{2} \mathrm{O}_{2}$ & $4 \pi$ & 437 \\
\hline $\mathrm{Fe}_{\mathrm{c}_{2} \mathrm{O}_{2}}$ & 31 & 024 \\
\hline $\mathrm{CaO}$ & 62.8 & 66.52 \\
\hline $\mathrm{MgO}$ & 26 & 076 \\
\hline $\mathrm{SO}_{4}$ & 3.1 & 287 \\
\hline L.O.I. & 18 & 227 \\
\hline $\mathrm{C}_{2} \mathrm{~S}$ & 503 & 62 \\
\hline $\mathrm{C}-\mathrm{S}$ & 144 & 17 \\
\hline $\mathrm{C}_{3} \mathrm{~A}$ & 5.3 & ل \\
\hline $\mathrm{C}, \mathrm{AF}$ & 0.3 & - \\
\hline
\end{tabular}

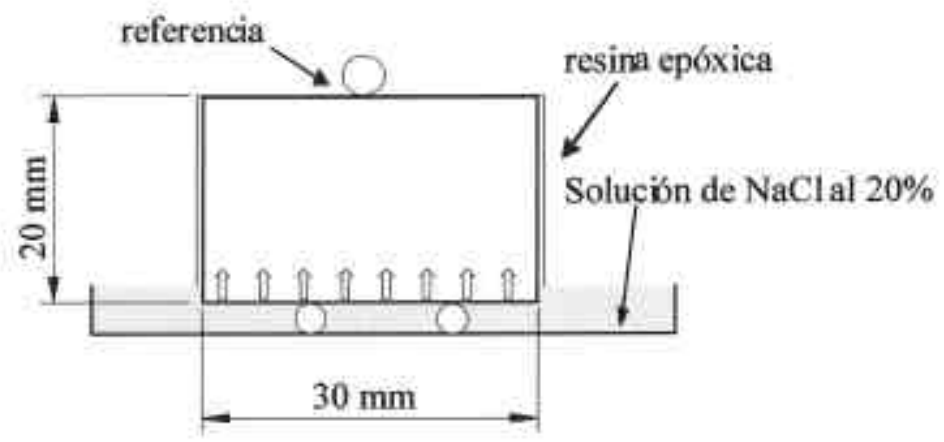

Figura 2. Arreglo utilizado para la prueba de absorción uniaxial de solución de $\mathrm{NaCl}$ y difusión en especimenes de mortero. Los experimentos de absorción capilar se llevan a cabo a la temperatura ambiente y los especimenes usados para difusión se mantuvieron dentro de una cámara ambiental a $38^{\circ} \mathrm{C}$

Se realizaron experimentos para determinar los tiempos de relajación $\mathrm{T}_{2}^{*}$ y $\mathrm{T} 1$ de ${ }^{1} \mathrm{H},{ }^{23 \mathrm{Na}}$ y ${ }^{35} \mathrm{Cl}$ en los especimenes estudiados. Los perfiles de distribución se obtuvieron con la técnica SPRITE utilizando un magneto superconductor Nalorac (Martinez, CA) de 2.4 Tesla, $32 \mathrm{~cm}$ de diámetro interior, con un micro-gradiente de $8 \mathrm{~cm}$ de diámetro interior enfriado con agua. Se utilizó un emisor/receptor de radio frecuencia tipo jaula (Morris Instruments, Ottawa) para la determinación de ${ }^{1} \mathrm{H}$, así como un nuevo diseño para el caso de ${ }^{23} \mathrm{Na}$ y ${ }^{35} \mathrm{Cl}$. La consola del espectrómetro fue una Tecmag (Houston, TX) Apolo. El amplificador de radio frecuencia fue el modelo 3445 (Brea, CA) de $2 \mathrm{Kw}$ de potencia.

Los especimenes usados para la absorción capilar de solución de $\mathrm{NaCl}$ se colocaron en un recipiente poco profundo y se permitió absorber la solución en un extremo. La concentración de $\mathrm{NaCl}$ en el recipiente fue de $200 \mathrm{~g} / \mathrm{L}$, la cual es aproximadamente seis veces el de $\mathrm{NaCl}$ en agua de mar. Se 


\section{P.F. de J. Cano-Barrita, B. J. Balcom y T.W. Bremner}

realizaron mediciones con la técnica SPRITE para observar la penetración de ${ }^{23} \mathrm{Na}\left(\mathrm{Na}^{+}\right), y$ ${ }_{35} \mathrm{Cl}\left(\mathrm{Cl}^{-}\right)$. Debe mencionarse que ${ }^{37} \mathrm{Cl}$ no contribuyen a la señal porque su frecuencia de resonancia es diferente de la de ${ }^{35} \mathrm{Cl}$. La abundancia natural de cada uno es $24.47 \%$ y $75.53 \%$, respectivamente.

Un arreglo similar al mostrado en figura 2 fue usado para los experimentos de difusión de $\mathrm{Na}^{+}$y $\mathrm{Cl}^{-}$en especimenes de mortero de cemento Pórtland blanco. Dos especimenes idénticos saturados con agua fueron puestos en contacto con una solución de $\mathrm{NaCl}$ al 20\%. Las medidas de SPRITE uni-dimensionales se realizaron semanalmente por un período de seis semanas.

En cada experimento, una referencia conteniendo solución de $\mathrm{NaCl}$ se colocó sobre el espécimen para permitir escalar los perfiles y compensar por variaciones pequeñas en la sensibilidad del instrumento (Figura 2).

\section{Resultados y discusión}

\author{
Tiempos de relajación
}

Los tiempos de relajación $\mathrm{T}$ y $\mathrm{T}_{2}{ }^{*}$ para ${ }^{1} \mathrm{H}$, ${ }^{23} \mathrm{Na}$ y $35 \mathrm{Cl}$ en mortero a 72 horas de iniciado el experimento, se muestran en el cuadro 2. Ambos tiempos son menores de $5 \mathrm{~ms}$, lo que hace a SPRITE una técnica requerida para obtener imágenes de estas especies. Los tiempos de relajación $\mathrm{T} 1 \mathrm{y} \mathrm{T}_{2}^{*}$ son exponencial simple, salvo los $\mathrm{T} 2{ }^{*}$ para el sodio y cloruro que tienen dos componentes. Note que los tiempos de relajación en mortero que contiene cemento ordinario son del mismo orden de magnitud comparado a los especimenes que contienen cemento blanco. Esto indica que los mecanismos de relajación dominantes en ${ }^{23} \mathrm{Na}$ y ${ }^{35} \mathrm{Cl}$ son diferentes a los que se tienen en la relajación de ${ }^{1} \mathrm{H}$ del agua, cuya relajación es rápida y no puede observarse fácilmente con el equipo disponible en el laboratorio.

Cuadro 2. Tiempos de relajación $\mathrm{T}_{1}$ y $\mathrm{T}_{2}{ }^{*}$ para $1 \mathrm{H}, 23 \mathrm{Na}$ y $35 \mathrm{Cl}$ en especimenes de mortero fabricado con cemento Pórtland blanco y ordinario

\begin{tabular}{|c|c|c|c|c|}
\hline \multirow[t]{2}{*}{ Núdeo } & \multicolumn{2}{|c|}{$\begin{array}{l}\text { Mortero de cemento } \\
\text { Portland blanco }\end{array}$} & \multicolumn{2}{|c|}{$\begin{array}{l}\text { Mortero de cemento } \\
\text { Porttland ordinario }\end{array}$} \\
\hline & $T_{1}(m s)^{1}$ & $T_{2}{ }^{\prime}(u s)^{1}$ & $\mathrm{~T}_{1}(\mathrm{~ms})^{1}$ & $T_{2}^{*}$ (us) ${ }^{1}$ \\
\hline${ }^{1} \mathrm{H}$ & 4.8 & 300 & ND & ND \\
\hline${ }^{23} \mathrm{Na}$ & 3.5 & $\begin{array}{l}147^{2} \\
755^{-3}\end{array}$ & 1.5 & 132 \\
\hline${ }^{35} \mathrm{Cl}$ & 2.2 & $\begin{array}{l}184^{2} \\
917^{3}\end{array}$ & 1.4 & 270 \\
\hline
\end{tabular}

1 Valores a 72 horas de absorción. Todos los tiempos de relajación ajustados tuvieron incertidumbres bajo $10 \%$, y en algunos cascos mucho menos.

2 La componente corta de los tiempos de relajación contribuye $31 \%$ y $29 \%$ de la magnitud total en ${ }^{36} \mathrm{Cl} \mathrm{y}{ }^{23} \mathrm{Na}$, respectivamente.

3 La componente larga del tiempo de relajación contribuye $69 \%$ y $71 \%$ de la magnitud total en ${ }^{36} \mathrm{Cl} \mathrm{y}^{23} \mathrm{Na}$, respectivamente. 
Imaginología por resonancia magnética y la determinación de cloruro y sodio en mortero de ...

\section{Difusión de ${ }^{23} \mathrm{Na}$ y ${ }^{35} \mathrm{Cl}$ en mortero de cemento Pórtland blanco}

Las figuras 3 y 4 muestran la distribución de $\mathrm{Cl}^{-}$y Na${ }^{+}$, respectivamente, obtenida en dos especimenes idénticos preparados con cemento Pórtland blanco. Nótese que los resultados en ambas figuras son muy similares, como es de esperarse. En éstas figuras puede observarse que la concentración en la cara en contacto con la solución de $\mathrm{NaCl}$ aumenta durante la primera semana de exposición. Después de este período, la concentración en la cara permanece casi constante y sólo la profundidad de penetración aumenta con el tiempo.

Los coeficientes de difusión efectivos fueron estimados ajustando los datos que corresponden a los perfiles obtenidos después de tres semanas, mostrados en figuras 3 y 4 a la ecuación 2, que es la solución a la segunda ley de difusión de Fick (Cranck, 1975). Normalmente, se ajustan perfiles de concentración del cloruro a esta ecuación para encontrar los Co y los Deff (Crack, 1975, Andrade et al., 2000).

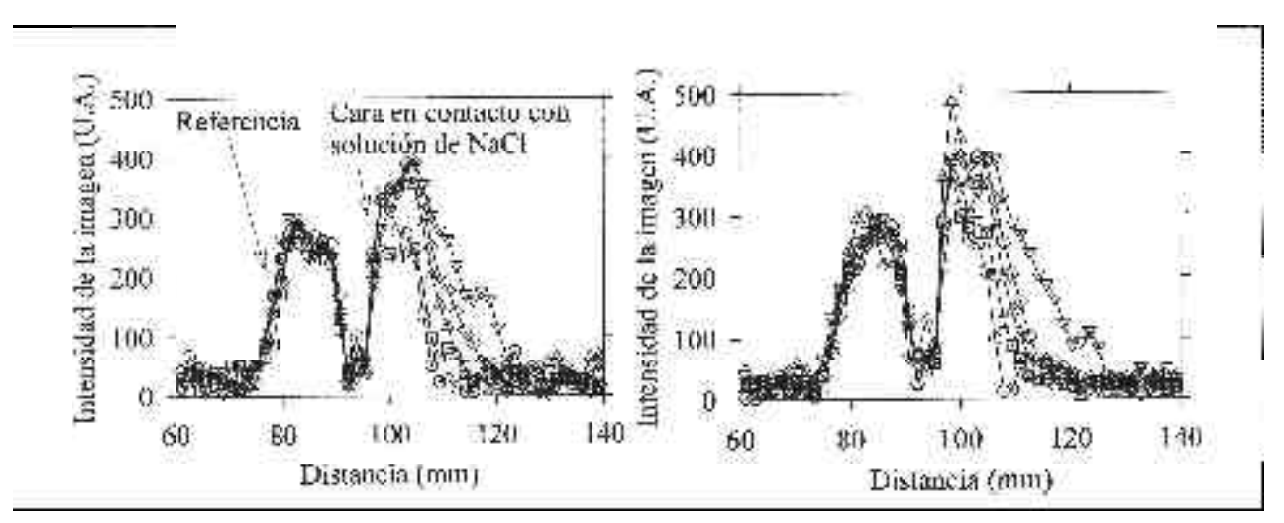

Figura 3. Distribución de ${ }^{35} \mathrm{C} 1$ en dos especimenes idénticos de mortero blanco $w / c=0.60$ a 1 semana $(\mathrm{O}), 2$ semanas $(\square), 3$ semanas $(\triangle), 4$ semanas $(\diamond)$ y 6 semanas $(\nabla)$. Los parámetros usados para la técnica SPRITE fueron: $F O V=200 \mathrm{~mm}$, resolución nominal $=1.6 \mathrm{~mm}, \mathrm{Gmax}=25.6 \mathrm{G} / \mathrm{cm}, \mathrm{TR}=2 \mathrm{~ms}$, elt $p=150 \mu \mathrm{s}$, ángulo $\alpha=44^{\circ}$, scans $=8192$ y tiempo de adquisición $=20.7$ minutos

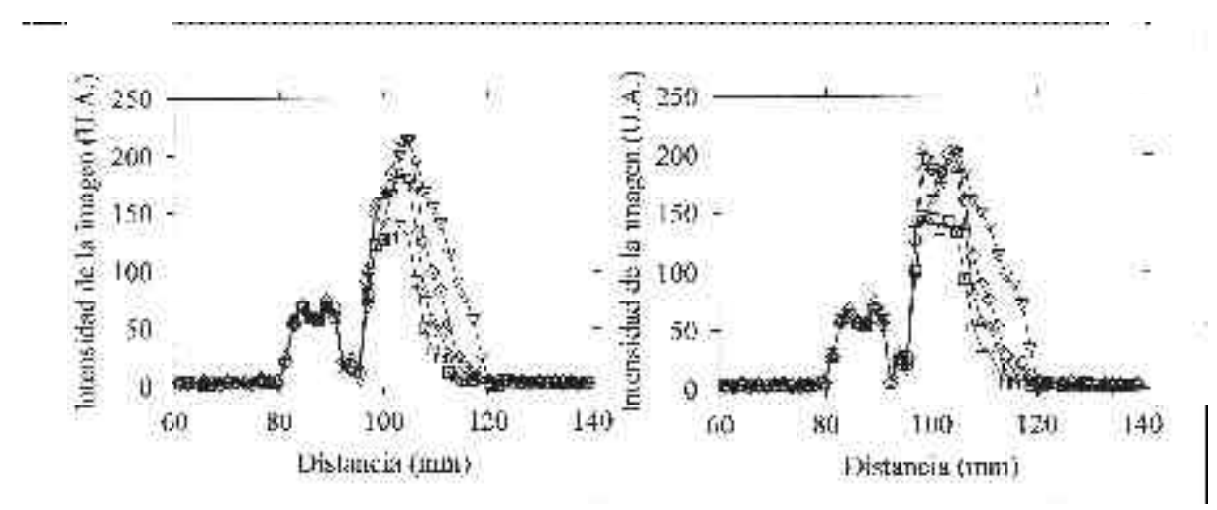

Figura 4. Distribución de ${ }^{3} \mathrm{Na}$ en dos especimenes de mortero blanco $w / c=0.60$ a 1 semana $(\mathrm{O}), 2$ semanas $(\square), 3$ semanas $(\triangle), 4$ semanas $(\diamond)$ y 6 semanas $(\nabla)$. Los parámetros usados para la técnica SPRITE fueron: FOV=200 mm, resolución nominal $=1.6 \mathrm{~mm}, \mathrm{Gmax}=9.5 \mathrm{G} / \mathrm{cm}, \mathrm{TR}=2 \mathrm{~ms}, \mathrm{el} t \mathrm{t}=150 \mu \mathrm{s}$, ángulo $\alpha=49^{\circ}$, scans $=2048$ y tiempo de adquisición=7.4 minutos 


$$
\frac{C(x, t)}{C_{0}}=1-\operatorname{erf}\left(\frac{x}{\sqrt{4 D_{e f f} t}}\right)
$$

El resultado fue Deff $=1.7 \times 10^{-11} \mathrm{~m}^{2} / \mathrm{s}$ para ambos especimenes. El coeficiente de difusión correspondiente para sodio fue $D_{\text {eff }}=$ $1.5 \times 10^{-11} \mathrm{~m}^{2} / \mathrm{s}$. De la literatura, el coeficiente de difusión de cloruro para concreto con relación $\mathrm{a} / \mathrm{c}=0.60$ y curado en húmedo durante 28 días es aproximadamente $1.8 \times 10^{12}$ $\mathrm{m}^{2} / \mathrm{s}$ (Nilsson et al ., 2000), y para concreto con relación $\mathrm{a} / \mathrm{c}=0.58$ y curado en húmedo $\mathrm{du}$ rante 28 días es aproximadamente $5.2 \times 10^{11}$ $\mathrm{m}^{2} / \mathrm{s}$ (Bamforth, 1993). Los valores de estos autores sólo sirven para ilustrar el orden de magnitud, porque los especimenes usados para resonancia magnética se hicieron de mortero y las condiciones de curado también fueron diferentes.

\section{Absorción capilar de una solución de $\mathrm{NaCl}$ en mortero de cemento Pórtland ordinario}

Las figuras 5 y 6 muestran los perfiles de cloruro y sodio, respectivamente, obtenidos en especimenes de mortero elaborado con cemento ordinario durante absorción capilar de solución de $\mathrm{NaCl}$. Este es un resultado muy importante de los experimentos, ya que es posible obtener perfiles del cloruro libre y distribución de sodio en mortero que contiene cemento ordinario

Esto hace de la resonancia magnética una poderosa herramienta para determinar la distribución de cloruros y la profundidad de penetración en concreto ordinario en laboratorio y en corazones extraídos de estructuras en campo. Además, su naturaleza no destructiva permite monitorear el mismo espécimen sin perturbar los procesos que ocurren dentro del mismo.

Otra área importante de aplicación de esta técnica podrá ser la validación de modelos matemáticos que predicen el ingreso de cloruros en concreto (Mangat, 1994, Marchand, 2001). Aunque no se probaron especimenes de concreto que contienen aditivos minerales, no representará un problema, dado que la cantidad de hierro no es tan importante cuando los cloruros son los núcleos de interés.

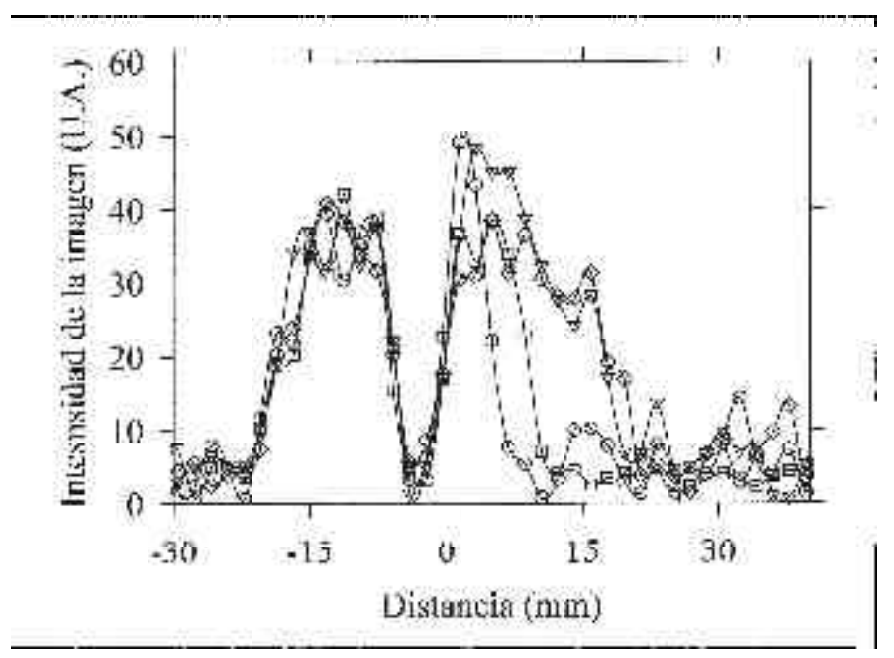

Figura 5. Distribución de ${ }^{35} \mathrm{C} 1$ en mortero elaborado con cemento ordinario $a / c=0.60$ a $3 h(\mathrm{O}), 9 h(\square), 2$ días $(\nabla)$ y 5 días $(\diamond)$. Los parámetros usados para la técnica SPRITE fueron: FOV=100 $\mathrm{mm}$, resolución nominal $=1.6 \mathrm{~mm}, \mathrm{G} \max =25.6 \mathrm{G} / \mathrm{cm}, T R=2 \mathrm{~ms}$, el $t_{p}=300 \mu \mathrm{s}$, ángulo $\alpha=44^{\circ}$, scans=8192 y tiempo de adquisición=20.7 minutos 


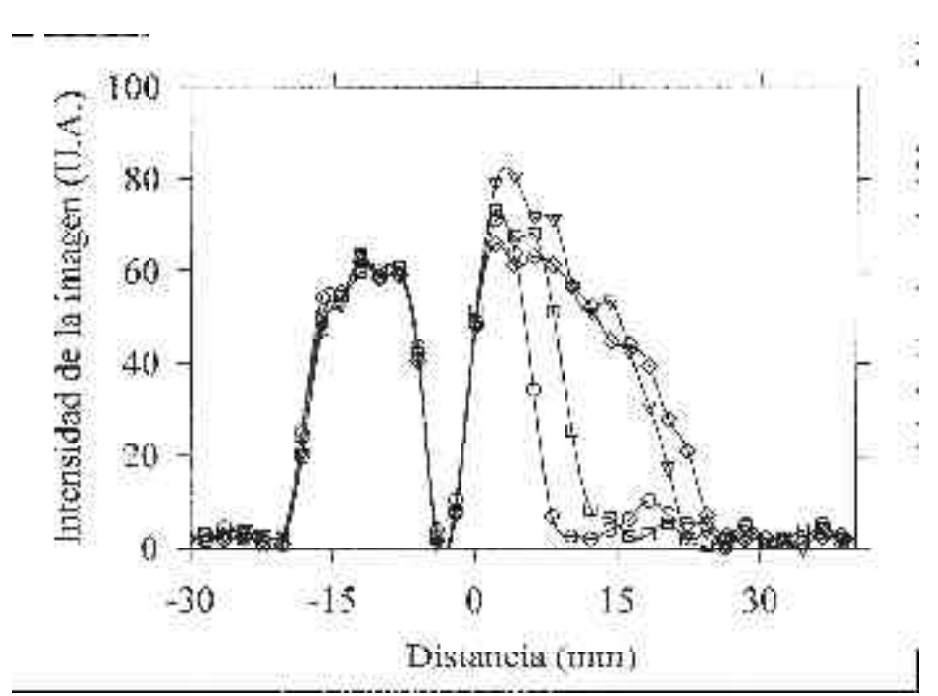

Figura 6. Distribución de ${ }^{2} \mathrm{Na}$ en mortero elaborado con cemento ordinario $a / c=0.60$ a $2.5 \mathrm{~h}(\mathrm{O}), 8 \mathrm{~h}(\square)$, 2 días $(\nabla)$ y 5 días $(\diamond)$. Los parámetros usados para la técnica SPRITE fueron: $F O V=100 \mathrm{~mm}$, resolución nominal $=1.6 \mathrm{~mm}, \mathrm{Gmax}=9.5 \mathrm{G} / \mathrm{cm}, T R=2 \mathrm{~ms}$, el t $p=300 \mu \mathrm{s}$, ángulo $\alpha=49^{\circ}$, scans $=2048$ y tiempo de adquisición=7.4 minutos

\section{Conclusiones}

La técnica SPRITE desarrollada en el Centro de Resonancia Magnética de UNB puede utilizarse para determinar la distribución de cloruros, sodio, y otros núcleos de interés en investigación relacionada con materiales basados en cemento. La determinación no-destructiva de la distribución del cloruro libre en mortero que contenga no sólo cemento blanco, sino también cemento ordinario, es posible. Los resultados obtenidos con cemento del Pórtland ordinario indican que el contenido de hierro no es un factor limitante para determinar perfiles de cloruros, por lo tanto, pueden utilizarse también especimenes de concreto extraído en campo.

Mejoras en el equipo y en las técnicas tienen lugar en el Centro de Resonancia Magnética de la Universidad de New Brunswick, lo cual permitirá aumentar la sensibilidad de las mediciones y probablemente se podrán utilizar concentraciones de las solución de $\mathrm{NaCl}$ similar a las del agua de mar (aproximadamente $3.5 \%$ ).
Debe mencionarse que aun cuando la determinación de la distribución de cloruros es no-destructiva, existe la necesidad de extraer una muestra de la estructura.

Trabajo futuro será necesario para establecer una correlación de las medidas realizada usando resonancia magnética y la cantidad de cloruros determinadas por métodos estándar de la ASTM.

\section{Agradecimientos}

Balcom B.J. agradece los apoyos de NSERC para operación y equipo, así como de la NSERC Steacie Fellowship 2000-2002. También agradece al programa Canadá Chair. Cano-Barrita P.F. de J., agradece el apoyo financiero recibido del Instituto Politécnico Nacional de México y el CONACyT México.

\section{Referencias}

Andrade C., Sagrera J.L. and Sanjuan M.A. (2000). Several Years Study on Chlo ride Ion Pene tra tion in to Concrete 


\section{P.F. de J. Cano-Barrita, B. J. Balcom y T.W. Bremner}

Exposed to Atlantic. Proceedings of the $2{ }^{\text {nd }}$ Inter na tional RILEM Work shop on Testing and Modeling the Chloride Ingress into Concrete, Paris, France, pp. 121-133.

Balcom B.J., MacGregor R.P., Beyea S.D., Green D.P., Armstrong R.L. and Bremner T.W. (1996). Single Point Ramped Imaging with T1 Enhancement (SPRITE). Journal of Magnetic Resonance, Series A 123, pp. 131-134.

Bamforth P. (1993). ConcreteClassification for R.C. Structures Exposed to Marine and Other Salt-Laden Environments. Structural Faults and Repair-93, Edinburg.

Cano F. de J., Bremner T.W., McGregor R.P. and Balcom B.J. (2002). Magnetic Resonance Imaging of ${ }^{1} \mathrm{H},{ }^{23} \mathrm{Na}$, and ${ }^{35} \mathrm{Cl}$ Penetration in Portland Cement Mortar. Cement and Concrete Research, 32 (7), pp. 1067-1070.

Crank J. (1975). The Mathematics of Diffusion. 2nd Edn (Clar endon, Oxford).

Marchand J. (2001). Modeling the Behavior of Unsaturated Cement Systems
Exposed to Aggres sive Chem ical Environments. Materials and Struc tures, 34, pp. 195-200.

Mitrovic V.F., Sigmund E.E. and Halperin W.P. (2001). Progressive Saturation NMR Relax ation. Phys ical Review B , 64, pp. 1-7.

Nilsson L., Andersen A., Luping T. and Utgenannt P. (2000). Chlorides Ingress Data from Field Expo sure in a Swedish Road Envi ron ment. Proceed ings of the 2ndInternational RILEM Work shop on Testing and Modeling the Chloride Ingress into Concrete, Paris, France, pp. 69-83.

Otsuki N., Nagataki S. and Nakashita K. (1992). Evaluation of $\mathrm{AgNO}_{3}$ Solution Spray Method for Measurement of Chloride Penetration into Hardened Cementitious Matrix Materials. ACI Materials, 89 (3), pp. 587-592.

Pel L., Kopinga K. and Kaasschieter E.F. (2000). Saline Absorption in CalciumSilicate Brick Observed by NMR Scanning. Journal of Physics D; Applied Physics, 33, pp. 1380-1385.

\section{Semblanza de los autores}

P. Felipe de Jesus Cano-Barrita. Es miembro del Amer ican Concrete Insti tute (ACI) y del Instituto Mexicano del Cemento y del Concreto (IMCYC). En 2003, obtuvo su doctorado en ingeniería civil en la Universidad de New Brunswick, Canadá. Actualmente es investigador en el CIIDIR Oaxaca del Instituto Politécnico Nacional de México y miembro del SNI nivel 1. Su investigación se relaciona con el uso y durabilidad del concreto ordinario y de alto desempeño en climas cálidos, uso de polímeros naturales, puzolanas artificiales, concreto autoconsolidante, así como desarrollo y aplicación de ferrocemento en el diseño y construcción de estructuras prefabricadas ligeras.

Theodore W. Bremner. Es miembro del ACI, así como profesor investigador honorario de ingeniería civil en la Universidad de New Bruns wick, Canada. Fue pres i dente del Capítulo Atlántico del ACI. Recibió la medalla ACI Cedric Willson por su investigación en concreto ligero y fue presidente de los comités ACI 213-Lightweight Aggre gate Concrete y ACI 122-Energy Conser va tion.

B.J. Balcom. Es profesor investigador del departamento de física y director del Centro de Resonancia Magnética de la Universidad de New Bruns wick. Recibió una NSERC Steacie Fellow ship (2000-2002) y Canada Research Chair in MRI of Materials. Sus principales intereses en investigación son el desarrollo de técnicas de imaginología por resonancia magnética y su aplicación a ciencia de materiales. 